\title{
The economics of post-doc publishing
}

\author{
William W. L. Cheung* \\ Sea Around Us Project, Fisheries Centre, the University of British Columbia, 2202 Main Mall, Vancouver, \\ British Columbia V6T 1Z4, Canada
}

\begin{abstract}
The use of bibliometrics (e.g. citation index and impact factors) in measuring academic research performances can affect the publication strategy of researchers. Such effects may be particularly potent to young researchers who are at a transitional stage of their academic career. This paper explores how bibliometrics affect the publication strategy from a point of view of a post-doctoral fellow, with analogy and explanation from simple economic theories. A major objective of postdoctoral fellowship is preparation for an academic career, which includes building up publication records and academic reputation; both are related to bibliometrics and publications. The need to achieve these objectives in the relatively short time period of the fellowship is analogous to putting a high discount rate on the benefits from future publications and networking. This may affect the researcher's choice of focus and journal in which to publish. Specifically, ethnic minorities in the English-dominated science community may need to develop special publication strategies. My experiences of being a post-doc in the field of fisheries science are drawn upon for illustration. Excessive focus on building publication records may lead to over-bias towards choosing high-impact journals for publication, violation of publication ethics, and unbalanced development of writing skills. It is important for young scientists to recognize the multitude of objectives of our academic works and try to achieve a balanced portfolio of these objectives.
\end{abstract}

KEY WORDS: Post-doctoral fellow · Academic publishing · Impact factor · Academic career • Discount rate

\section{INTRODUCTION}

The validity of measuring research performance through publication records of a researcher and number of citations of published papers has long been the focus of considerable discussion and debate in the academic world (e.g. Garfield 1979, van Raan 2005). Specifically, researchers are concerned about the increasing use of these indices in job promotion, appointment and performance evaluation. The uses of these indices have obvious pros and cons. (e.g. Garfield 1979, Seglen 1997, Russell-Edu 2003, Bornmann \& Daniel 2005, Leimu \& Koricheva 2005, van Raan 2005, Pasterkamp et al. 2007), which I will not discuss here. As a young researcher (I started my post-doctoral fellowship 1 yr ago) trying to build a publication record and advance an academic career, I found that these 'rules of the game' (vis-à-vis Sindermann 1982) are affecting my publication strategy. In this paper, I attempt to explore the question of how they do this from the point of view of a post-doctoral fellow.

\section{'REWARDS' OF POST-DOCTORAL FELLOWSHIP}

For young scientists who want to pursue an academic career, publications and professional networks are some of the true rewards of being a post-doctoral fellow (besides, e.g., pay-checks and self-satisfaction, which are important as well). Post-doctoral fellowship is a transition from graduate study to a faculty or research position. Such a transition can expand 
one's research area and experience, build up a strong publication record and establish research networks, thus preparing oneself for the next step of the academic career. Particularly, junior faculty or other equivalent research positions in my field (fisheries/marine ecology) are often limited, while large cohorts of $\mathrm{PhD}$ graduates and post-docs are being produced each year. Thus, it becomes even more important for young scientists like myself to maximize outputs from their post-doctoral fellowship in order to get their job application to the top of the candidate list.

The 'rewards' of post-doctoral fellowship mentioned above can be expressed as a function of (1) number of publications and (2) number of colleagues who recognize my work and assess it positively. The importance of the former is obvious as the number of publications can indicate academic productivity and ability to deliver. Also, I found that publishing is one of the best ways to introduce my name to other researchers - an essential step to my second point (the 'name' factor relates to something that I will come back to in a later section). Clearly, networking is very important to job-seekers in the academic world. Institutes often target a specific person before they publicize a job opening. Even if a position is completely open, it is understandable that, out of the long list of applicants, the selection committee may take a better look at applications from candidates whom they know personally, through their publications or recommendations from peers. Thus, 'reputation' increases the chances of, at least, being short-listed in a job application. The importance of reputation in the scientific community has long been recognized by sociologists of science (e.g. Merton 1988). Following from this, establishing a good reputation in academia should be considered as a major achievement from the postdoctoral experience.

\section{ACADEMIC QUALITY AND RECOGNITION TIMING}

How does research quality come into the equation? Ideally, reputation in the scientific community should be closely correlated with the quality of the research and publications. However, reputation is also affected by the inherent high discount rate on the rewards or academic outputs from the post-doctoral experience. In economics, discount rate $(\delta)$ determines the present value of a flow of benefits. With higher $\delta$, benefits to be obtained in the future will be valued less at the present time (Fig. 1). For example, \$100 obtained after 10 yr would only be valued as about $\$ 62$ at present, with a discount rate of $5 \% \mathrm{yr}^{-1}$, or $\$ 39$ with a $10 \% \mathrm{yr}^{-1}$ discount rate. Discount rate may be negatively related

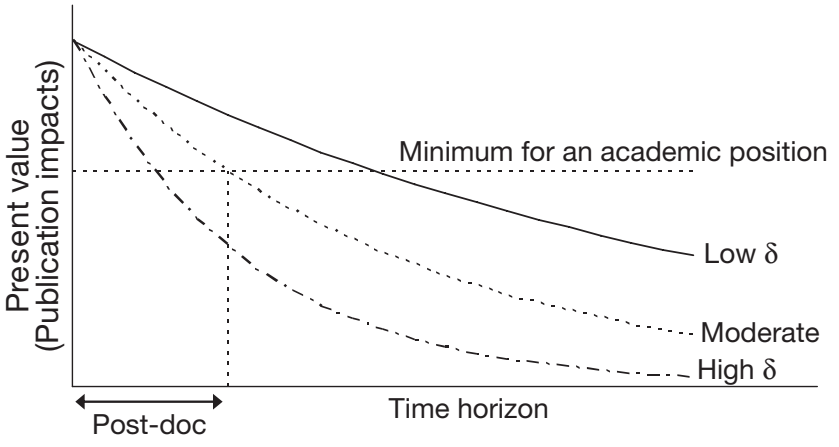

Fig. 1. Future benefits that are valued at the present time under 3 different levels of discount rate $(\delta)$ : low, moderate and high. Publications that are recognized by peers in the distant future are perceived as low value from the point of view of a post-doc

to the time frame of the project being valued (Frederick et al. 2002). Thus, in the context of publishing, having a short time-horizon of 2 to $3 \mathrm{yr}$ of an average post-doctoral fellowship means that my works need to be recognized as much as possible within this short time period. Then, my résumé can stand out from other colleagues while competing for the same job. This implies that there is a high discount rate on academic outputs of post-docs, and works that are recognized in the more distant future can be largely discounted. For instance, a publication that is read and recognized by 100 colleagues in 2 yr may be perceived as far more 'valuable' than one that is recognized by 200 colleagues in 4 years' time. The discount rate on academic output may gradually decrease along the academic career from post-doc to assistant, associate and full professorship as the frequency or importance of regular academic assessment decrease.

The high discount rate on post-doc publications may have led me to prefer publishing quickly (in journals with fast turn-around times) and in high-impact journals. Works presented in high-impact journals are not necessarily of superior quality than others (Seglen 1997; see Nature Editorial 2005). In fact, many papers published in specialized journals with relatively lower impact factors are of excellent quality; thus, a journal's impact factor does not necessarily reflect the quality of their papers (Garfield 1996, Seglen 1997, Russell-Edu 2003). However, high-impact journals may be read regularly by more people and provide more opportunities for the research to be featured in public media. Moreover, their publication rate is fast. Thus, with the perception that research can be more widely known if published in a high-impact journal, publishing in such journals is especially attractive to young scientists. 


\section{THE ‘NAME’ FACTOR}

Publishing is one of the best ways to introduce my name and work widely to fellow colleagues - provided that readers of my papers can remember my name. In my field and most other disciplines in science, English is the dominant language for publishing (Ammon 2001). Being a Chinese person from Hong Kong, my original name was, of course, fully Chinese. I was also given an 'official' English name translated by the government from the Cantonese (the Chinese dialect spoken in Hong Kong) pronunciation of my Chinese name (i.e. Wai Lung Cheung). However, non-Chinese speaking people often have problems pronouncing, spelling or even remembering my Cantonese-translated name, and this is true even for colleagues with whom I have worked for years. This is understandable as I also sometimes find it difficult to remember non-Chinese names. This corroborates with the finding that publications with non-English author names are more frequently mis-cited than those with English names (Kotiaho et al. 1999) and may explain why citations of papers from non-English-speaking countries were less than expected when compared to those from English-speaking countries (May 1997, Kotiaho 1999). The 'name' factor may have broader implications, as people with non-English ethnic names are shown to be disadvantaged in employment in Englishdominant societies such as the UK and New Zealand (Wilson et al. 2005), although its generalization to academic employment has not been demonstrated. One of my mentors also suggested that the 'name' factor was one of the cultural factors that could become a disadvantage to a non-English person in Englishdominated communities, and that I should adjust my name to account for this.

Thus, in recent years, I changed from using my Cantonese-translated first name (Wai Lung) to my English name (William) in scientific publications and academic communications, while keeping the Cantonese name as a middle name. Such changes may be seen as 'caving in' to the dominant culture. However, when Hong Kong was under British colonization (until 30 June 1997), most students in Hong Kong were asked to acquire an English name. Hence, the use of an English name is psychologically easy for me to accept. On the other hand, this may be considered a violation of one's culture to some colleagues. Thus, I am just sharing my experience, without advocating its adoption by others.

\section{IMPLICATIONS}

The high discount rate of post-doc publishing has implications beyond the choice of journals in which to publish. Firstly, it may affect the choice of research topics. The rejection rates of high-impact journals are extremely high and good work does not guarantee acceptance. 'Hot' topics are favored by these journals. Thus, young scientists may prefer research topics that have high potential impact. This was a factor that I considered when I was choosing from a few options for a post-doctoral fellowship. However, this may create a false impression that researchers tackling hot topics are superior to others.

Secondly, to increase the chance of publishing in high-impact journals, junior scientists may be inclined to let their supervisor or senior researchers be the lead authors of their work. Articles published in high-impact journals are highly concise and focused. Senior colleagues are generally more experienced and skillful in writing such research papers. However, having senior colleagues write papers based on a junior's works is unethical and should be strongly discouraged. Such practice also reduces the opportunity for the junior scientists to gain publishing experience and improve their writing skills. It would be better to ask your supervisor or senior colleagues to help and guide the writing instead of leading it. I am glad that my supervisor understands well that young scientists are more in need of such experience and has encouraged me to lead and write papers as much as possible.

Good scientific writing requires excellence in writing different styles of papers. This includes writing articles that target high-impact journals, more specialized journals, conference proceedings and book chapters, as well as popular articles. Thus, a more balanced portfolio of writing experience should provide more benefits to post-docs. Moreover, young scientists may even develop bad writing habitats (e.g. exaggerating implications of findings, over-simplifying analyses and conclusions, ignoring caveats) if an excessive desire to publish in high-impact journals skews their scientific judgement or publication ethics.

\section{TRADE-OFFS IN POST-DOC PUBLISHING}

An ideal post-doctoral experience should include a balanced portfolio of objectives. So far, this paper has focused on a single objective in post-doc publishing, i.e. to secure a decent job. However, I believe that researching and publishing contribute to a wider range of objectives, such as satisfaction of curiosity and serving the public good, which may not be directly related to citation measures. However, given limited resources that can be dedicated to the postdoctoral experience (e.g. time), trade-offs will be needed in achieving different objectives, i.e. achieving more of any one objective will reduce the achieve- 
ment of the others. On the other hand, 'win-win' solutions can be achieved, i.e. large benefits from most objectives can be obtained simultaneously, while large compromises with any objectives may not be necessary. However, there may be exceptions, for example, when the research challenges an established paradigm, or has large potential impacts that are not recognized at present. Such research may have difficulty getting published and, if it does get published, will have less immediate impact. I cannot offer any definite answer as to how this can be resolved, but suggest that we should keep reminding ourselves of the need to recognize the importance of the portfolio of objectives in our post-doctoral experience.

\section{CONCLUSIONS}

The use of publication counts and number of citations to assess academic performance do affect the publishing strategy of young scientists. Particularly, at the stage of being a post-doc, to compete for the limited number of junior faculty or equivalent research positions, one would try to maximize the number of publications and their impacts in a short time-horizon (which refers to the number of colleagues who recognize one's work) - analogous to putting a high discount rate on academic output. This may have implications for the development of academic careers. It is important for young scientists to recognize the multitude of objectives of our academic work besides making an impressive résumé.

Acknowledgements. I thank D. Pauly for the encouragement and advice in writing this paper. I am grateful to the editors of this Theme Section for their editing and comments. This is a contribution of the Sea Around Us Project supported by funding from the Pew Charitable Trusts.

Editorial responsibility: Howard Browman, Storebø, Norway and Konstantinos Stergiou, Thessaloniki, Greece

\section{LITERATURE CITED}

Ammon U (ed) (2001) The dominance of English as a language of science: effects on other languages and language communities. Mouton de Gruyter, New York

Bornmann L, Daniel HD (2005) Does the h-index for ranking of scientists really work? Scientometrics 65:391-392

Frederick S, Loewenstein G, O'Donoghue T (2002) Time discounting and time preference: a critical review. J Econ Lit 40:351-401

> Garfield E (1979) Is citation analysis a legitimate evaluation tool? Scientometrics 1:359-375

> Garfield E (1996) How can impact factors be improved? Br Med J 313:411-413

Kotiaho JS (1999) Papers vanish in mis-citation black hole. Nature 398:19

Kotiaho JS, Tomkins JL, Simmons LW (1999) Unfamiliar citations breed mistakes. Nature 400:307

Leimu R, Koricheva J (2005) What determines the citation frequency of ecological papers? Trends Ecol Evol 20:28-32

> May RM (1997) The science wealth of nations. Science 275:793-796

> Merton RK (1988) The Mathew effect in science. II. Cumulative advantage and the symbolism of intellectual property. Isis 79:606-623

Nature Editorial (2005) Not-so-deep impact. Nature 435: 1003-1004

Pasterkamp G, Rotmans JI, de Kleijn DVP, Borst C (2007) Citation frequency: a biased measure of research impact significantly influenced by the geographical origin of research articles. Scientometrics 70:153-165

Russell-Edu W (2003) The impact factor: your job may depend on it-but do you know what it is? Cancer Futures 2: 171-176

Seglen PO (1997) Why the impact factor of journals should not be used for evaluating research. Br Med J 314:497-502

Sindermann CJ (1982) Winning the games scientists play. Plenum Press, New York

van Raan AFJ (2005) Measuring science. In: Moed HF, Glänzel W, Schmoch U (eds) Handbook of quantitative science and technology research: the use of publication and patent statistics in studies of $\mathrm{S} \& \mathrm{~T}$ Systems. Kluwer Academic Publishers, Dordrecht, p 19-50

Wilson MG, Gahlout P, Liu L, Mouly S (2005) A rose by any other name: the effect of ethnicity and name on access to employment. University of Auckland Business Review $7: 65-72$

Submitted: October 16, 2007; Accepted: November 18, 2007 Proofs received from author(s): December 7, 2007 